\title{
Dynamic interpretation of brittle fracture
}

\author{
P. Brož \\ Department of Concrete Structures and Bridges, \\ Czech Technical University, Czech Republic
}

\begin{abstract}
This paper presents constitutive simulations for cracked solids based on continuum damage mechanics. The aim is to demonstrate that the models can predict the experimentally observed effects in brittle materials. For a reference specimen, we have chosen a slant crack that is centrally located in a concrete panel, under step tensile pulse loading. Simultaneously, we observed the location of the damage and the tension - softening phenomena. We include a technique employing standard finite elements and a domain integral, $\hat{J}$, to set the dynamic stress intensity factor for a fast growing crack in both a linear elastic and a viscoelastic body.

Keywords: dynamic characteristic, energy release rate, failure, flow rule, internal variable, strain rate, sublevel crack.
\end{abstract}

\section{Introduction}

Precise predictions of the response of brittle materials require an understanding of their dynamic fracture characteristics. Many observed effects, like the strainrate phenomena, crack-tip damage, and tension softening, may not be elucidated by classical elastodynamic fracture mechanics. In the proposed study, the dynamic fracture of brittle materials is expounded with the use of a continuum damage simulation in accordance with the E.P. Chen model [1]. Concurrently, the material is approximated as a continuum with a random distribution of sublevel cracks. Mobilization of these cracks by the load leads to progressive damage to the material. In the continuum grade, the softening of the material moduli responds to damage accumulation. To indicate the practicality of this model, we investigated, the problem of an oblique crack located centrally in a concrete slab, and subject to the impact of a step tension impulse applied at the boundary of the panel. This version explains the dynamic fracture course as a 
continuous increase in damage while the damage is limited to the volume fraction of the material that has been tensile relaxed by multiple crack growth and interaction.

In the analysis of dynamic stress intensity factors with straight finite elements, a difficulty arises in extracting these factors from the numerically stipulated stresses and displacements. To avoid this inconvenience, a finite domain $J$ integral, standing for the dynamic energy release rate, was initiated according to [2], and it was employed to determine the dynamic stress intensity factors for abruptly loaded static cracks.

\section{CDM model parts}

The general continuum damage model has three fundamental segments:

a. A set of independent internal variables, $p_{k}$, which together with the infinitesimal strain tensor $\varepsilon$ (or the stress tensor $\sigma$ ) are assumed to specify uniquely the momentary state of the solid at a given point.

The internal variables may stand for a physical quantity or may be abstract in nature. They can be related to kinematic properties or to structural characteristics. For example, the vector $\mathrm{e}^{\mathrm{c}}$ in the smeared crack model is intended to represent the internal kinematics of cracks and n, the crack direction, is a structural internal variable. It should be noted that when a set of internal variables is selected, any other set, equivocally related to the first, is rigorously equivalent to it and, for this reason, can be employed instead of the first. This makes the physical interpretation of a given set of internal variables marginally ambiguous.

b. An equation set expressing the stress to the strain and to the internal variables:

$$
\sigma=\mathbf{S}\left(\varepsilon, \mathrm{p}_{\mathrm{k}}\right)
$$

In present-day thermodynamic formulations, eqn (1) is deduced from a free energy function that represents a scalar function to be determined instead of (1). As a rule, eqn (1) is assumed to be linear in the infinitesimal strain tensor.

c. A set of "flow rules", characterizing the manner in which the internal variables increase when loading proceeds. This is a delicate and essential point, since prescribing different flow rules to models having the same set of internal variables and the same structure for the stress strain relation, eqn (1), will lead to very different behaviour. Moreover, the flow rules must be consistent with the irreversibility condition posed by the Second Principle of Thermodynamics.

The flow rules may be particularized at many different grades of generality: A rather universal treatment for time-independent behaviour is to use one or more loading functions obtained by direct generalization of the theory of classical plasticity. For this purpose, internal forces $\mathrm{q}_{\mathrm{k}}$ conjugate with the 
internal variables must be chosen (they are readily obtained in a thermodynamic formulation), and a loading function $F\left(\mathrm{q}_{\mathrm{k}}\right)$ must be specified, so that for the region in which the characteristics are elastic (i.e.: $\mathrm{dp}_{\mathrm{k}}=0$ for any k) it holds:

and the associated flow rules are:

$$
\mathrm{F}\left(\mathrm{q}_{\mathrm{k}}\right) \leq 0
$$

$$
\mathrm{dp}_{\mathrm{k}}=\left(\partial \mathrm{F} / \partial \mathrm{q}_{\mathrm{k}}\right) \mathrm{d} \mu, \quad \mathrm{d} \mu \geq 0
$$

It is evident that a hierarchical organization of internal variables is possible, so that the primary set $p_{k}$ appearing in equation (1) is complemented by a secondary set of hardening-softening parameters entering the loading function (2).

Though this is a rather general formulation, it is not the only one possible, e.g. another is of multi-yield surface type. We can also generate restricted flow rules for particular loading instances, among which monotonic loading is the simplest and most effective.

\section{A simulation type}

Let us assume that the material is penetrated in the damage model by an array of randomly distributed cracks which propagate and interact with one another subject to tensile loading. The simulation does not endeavour to use each individual crack but rather handles the growth and interaction of the cracks as an internal state variable which stands for the damage accumulation in the material. This damage, $D$, is assumed to reduce the material stiffness according to the Budiansky and $\mathrm{O}^{\prime}$ Connell equations applicable to a random array of penny-shaped cracks in an isotropic elastic milieu

$$
\bar{K}=K(1-D)
$$

where $K$ and $\bar{K}$ mean, respectively, the bulk modulus for an undamaged and a damaged material. The damage is related to the damaged Poisson's ratio $\bar{v}$ and crack density parameter $C_{d}$ by means of the expression

$$
D=\frac{16\left(1-\bar{v}^{2}\right)}{9(1-2 v)} C_{d}
$$

The crack density parameter relates to the undamaged Poisson's ratio, $v$ and $\bar{v}$ via

$$
C_{d}=\frac{45}{16} \frac{(v-\bar{v})(2-\bar{v})}{\left(1-\bar{v}^{2}\right)[10 v-v(1+3 v)]}
$$

Accordingly, when the crack density parameter is known, the damaged Poisson's ratio can be determined from eqn (6) and the damage parameter is found from 
eqn (5). The crack density parameter gives information about cracking in a given volume, in that it is assumed to be proportional to the product of $N$, the quantity of cracks per unit volume, and $a^{3}$, the cube of the mean crack size in the volume element examined, i.e.

$$
C_{d} \sim N a^{3}
$$

As a rule, $N$ is expressed as a Weibull statistical distribution function stimulated by the present bulk strain measure $P / 3 \bar{K}$, where $P$ is the pressure or mean stress $P=\left(\sigma_{x x}+\sigma_{y y}+\sigma_{z z}\right) / 3$, in compliance with

$$
N=k\left(\frac{P}{3 \bar{K}}\right)^{m}
$$

In eqn (8), $k$ and $m$ are material constants to be specified from the strain-rate dependent fracture stress particulars. Since the size of the splinters is determined by the intersecting crack network within the concrete volume, the crack dimension is considered to be proportional to the splinter size. Consequently, the average crack size, $a$, is estimated from the nominal splinter diameter expression for dynamic fragmentation in a brittle material, being

$$
2 a \sim\left(\frac{\sqrt{20} K_{I c}}{\rho C_{s} \dot{\varepsilon}_{\max }}\right)^{2 / 3}
$$

where we have denoted

$$
\begin{aligned}
& \rho \ldots \ldots \ldots \text { mass density } \\
& C_{s} \ldots \ldots \ldots \text {. uniaxial wave velocity }(\sqrt{E / \rho}) \\
& E \ldots \ldots \ldots \text { Young's modulus } \\
& K_{I C} \ldots \ldots \ldots \text { fracture toughness of the material, and } \\
& \dot{\varepsilon}_{\max } \ldots \ldots \text { maximum measuring strain rate experienced by the } \\
& \quad \text { material in the course of the fracture process }
\end{aligned}
$$

The average crack size that has been activated by the load applied in the volume element under consideration results from eqn (9). Thus, when the strain rate is low, only large cracks have been activated and the material can only separate into a few large pieces. On the other hand, under high strain rates, smaller cracks would also have been activated and the specimen can break into many small fragments. The proportionality constants from eqns (7) and (8) can be absorbed into constant $k$. Hence, the additional material parameters for this constitutive model, aside from the commonly defined ones, are $k$ and $m$ as given in eqn (8). When bulk tension occurs in the material, we can at each time step calculate the damage parameter, $D$, from the above expressions. The stiffness is then degraded by the factor $(1-D)$. In this fashion, the post-damage responses of the material are represented. Note that the damage parameter, $D$, is an internal state variable 
which is evolutionary and irreversible in reality. In compression, it is assumed that the material characteristics are elastic/perfectly plastic.

\subsection{Dynamic material characteristics}

Constants $k$ and $m$ in the simulation are specific to the dynamic responses of the material considered. Representative values of concrete are listed in Table 1.

In order to evaluate constants $k$ and $m$ for the damage model, strain-rate-dependent tensile strength particulars for concrete in the range of strain rates of 1 to $100 / \mathrm{s}$ are required. These data can be estimated from the fracture toughness value via the equation

$$
\sigma_{\mathrm{c}}=\left(\frac{9 E K_{I c}^{2}}{16 Y^{2} C}\right)^{1 / 3} \dot{\varepsilon}^{1 / 3}
$$

Concurrently, $Y$ is a crack geometric shape factor, and $C$ means the shear wave velocity. Assuming that the sublevel cracks are penny-shaped, $Y$ amounts to 1.12. The results of this estimation are demonstrated in fig. 1. Constants $k$ and $m$ can be stipulated from the strain-rate dependent fracture stress. The quantities of $k$ and $m$ relevant to the issues in fig. 1 are derived to be $5.75 \times 10^{21} / \mathrm{m}^{3}$ and 6.0 , respectively.

Table 1: $\quad$ Typical concrete properties.

\begin{tabular}{|l|l|}
\hline Mass density & $2.4 \mathrm{Mp} / \mathrm{m}^{3}$ \\
Young's modulus & $20.7 \mathrm{GPa}$ \\
Poisson's ratio & 0.18 \\
Fracture toughness & $2.75 \mathrm{MPa} \sqrt{m}$ \\
Compressive strength & $27.6 \mathrm{MPa}$ \\
\hline
\end{tabular}

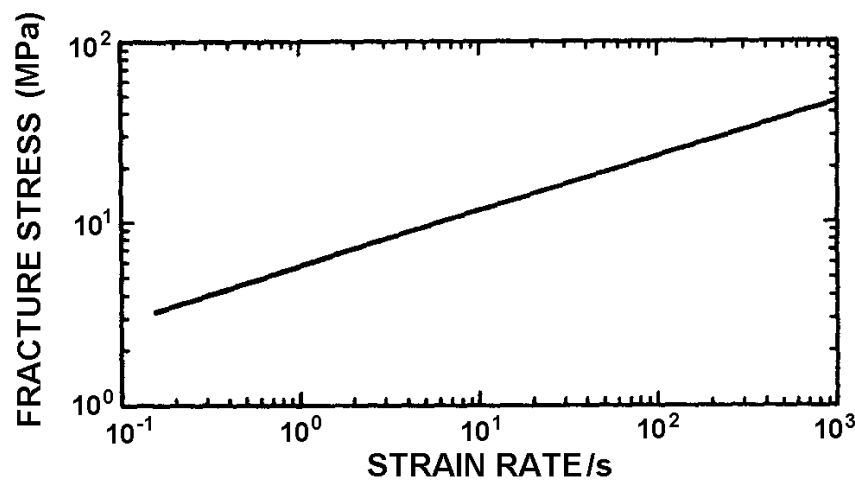

Figure 1: $\quad$ Strain rate versus fracture stress for concrete. 


\subsection{A panel weakened by a slant crack}

The structure is subject to uniform tension exerted at the top and bottom edges according to fig. 2, with plane strain conditions being considered in the investigated region. The load is applied in the form of a step function in time valued at $10 \mathrm{MPa}$. The finite element grid is demonstrated in fig. 3 .

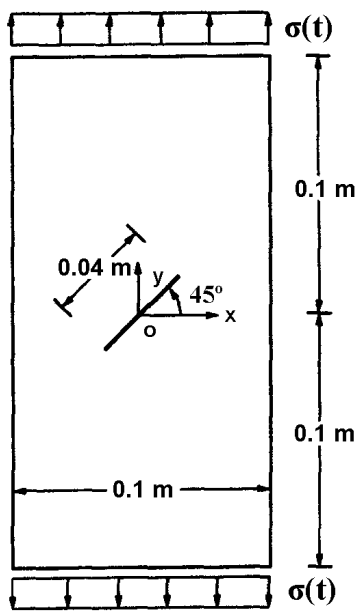

Figure 2: Example problem geometry.

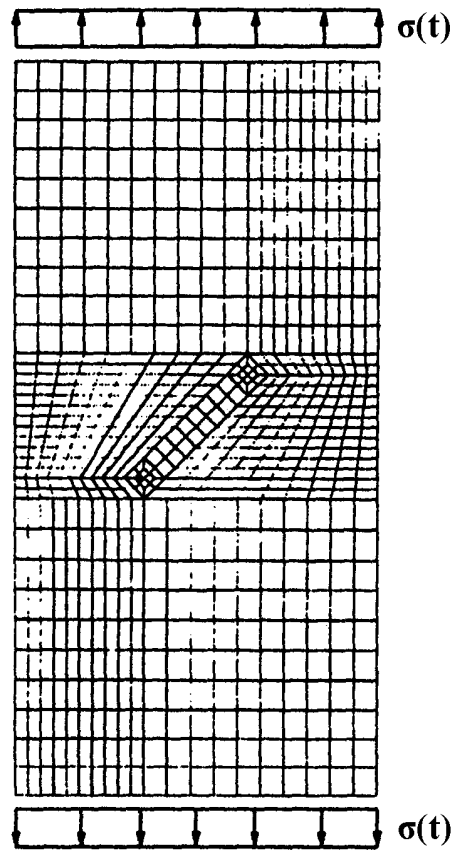

Figure 3: Finite element grid for the example problem. 
All tests were finalized at $0.4 \mathrm{~ms}$ because, at this time, a minimum of two loading and unloading cycles had appeared close to the elements round the crack tips. This is adequate time to explain some of the main traits in the damage simulation. The results were obtained from the PRONTO 2D code [3]. The crack surfaces are considered to be contact ones to prevent them from flipping over subject to compressive stresses from waves reflected from the boundaries. The mean stress against time plot for the element close to the lower crack tip in the case of both the elastic and damage models is indicated in fig. 4 . The position of this element is demonstrated in fig. 3, being a darkened square. This element, which is immediately ahead of the crack tip, is designated as Element 1 . The element whose location corresponds to Element 1 for the upper crack tip has similar behaviour and thus will not be shown. Because of the interactions between the stress waves and the bounding surfaces, this element will have multiple loading and unloading cycles. Because of the sublevel crack activation in the damage model, damage is being accumulated in the element. Therefore, the pressure was relaxed in the damage model and its magnitude began to decrease shortly after $0.05 \mathrm{~ms}$, notwithstanding that loading continues in the elastic mode. The damage time-history for this element is demonstrated in fig. 5 . This figure shows that the damage initially increases with time, following in essence the stress curve in fig. 4, and finally reaches an altitude of approximately 0.66 . Note that damage does not decrease after $t=0.05 \mathrm{~ms}$ even if there is unloading is owing to wave interactions. The damage accumulation advances irreversibly.

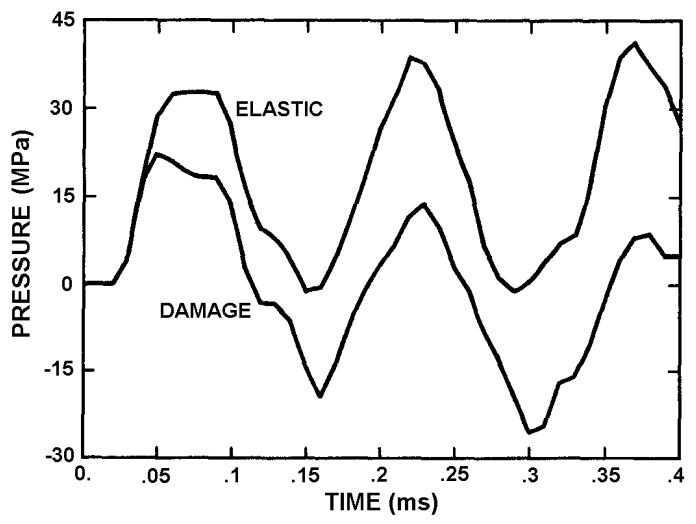

Figure 4: $\quad$ Time versus pressure at Element 1.

\section{Dynamic stress intensity factor for a rapidly growing crack}

The method employing the conventional elements and the $\hat{J}$-integral is applied to the problems of rapid crack propagation in elastic and also in viscoelastic solids. The energy release rate $\hat{J}$ has the form: 


$$
\hat{J}=-\lim _{\varepsilon \rightarrow 0} \lim _{\delta \rightarrow 0} \int_{\Gamma_{\text {end }}} T_{i} u_{i, 1} d \Gamma
$$

where $\Gamma_{\text {end }}$ stands for the rectangular path as demonstrated in fig. $5, T_{i}$ the fraction and $u_{i}$ the displacements. Note that the path $\Gamma_{\text {end }}$ should be contracted on the crack tip by permitting $\delta \rightarrow 0$ and then letting $\varepsilon \rightarrow 0$.

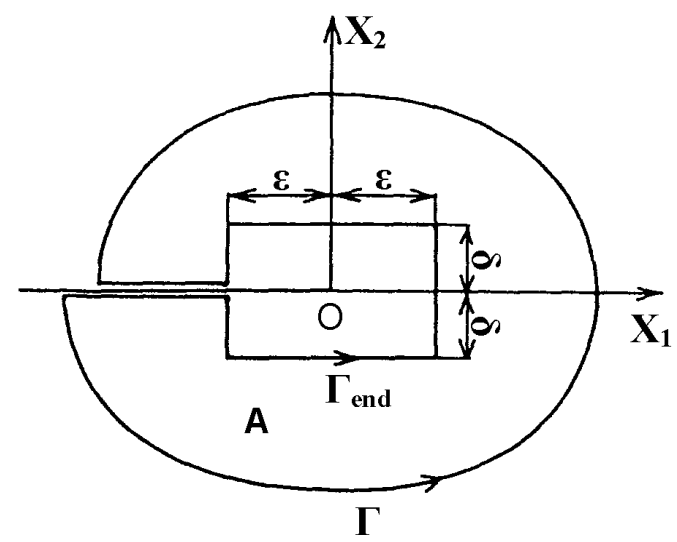

Figure 5: $\quad$ Infinitesimally small rectangular path $\Gamma_{\text {end }}$ and arbitrary path $\Gamma$

Let us assume a finite region round the crack tip, and let $\Gamma$ indicate the boundary of the region and A designate the area bounded by $\Gamma$ and $\Gamma_{\mathrm{d}}$ and the crack surfaces. Application of the divergence theorem to eqn. (4.1) gives a relation for $\hat{J}$ :

$$
\hat{J}=\lim _{\varepsilon \rightarrow 0} \lim _{\delta \rightarrow 0} \iint_{A}\left(\rho \ddot{u}_{i} u_{i, 1}+\sigma_{i j} \varepsilon_{i j, 1}\right) d A-\int_{\Gamma} T_{i} u_{i, 1} d \Gamma
$$

where $\varepsilon_{i j}$ represents the strain and dot (.) the time derivative. It is noted that the quantity of eqn. (12) is independent of the path $\Gamma$ for any standard of material response.

In the case of an elastic material, elastic strain energy density $\mathrm{W}_{\mathrm{e}}$ is given by the relation

$$
\mathrm{W}_{\mathrm{e}}=\frac{1}{2} \sigma_{i j} \varepsilon_{i j}
$$

Accordingly for eqn. (12) it holds:

$$
\hat{J}=\lim _{\varepsilon \rightarrow 0} \lim _{\delta \rightarrow 0} \iint_{A} \rho \ddot{u}_{i} u_{i, 1} d A+\int_{\Gamma}\left(W_{e} n_{1}-T_{i} u_{i, 1}\right) d \Gamma
$$

where $n_{i}$ implies the unit outward normal. For a fast growing crack, the integrand of the area integral in eqn. (14) is singular as $\mathrm{r}^{-2}$ (r: distance from the crack tip) 
and hence the value of the integral depends on the limiting process. Therefore, the limiting symbols should not be left out in eqn. (14), likewise in eqn. (12).

To reduce the order of the singularity in the integrand of the area integral, the following equation is taken away from eqn. (14)

$$
\hat{J}=\lim _{\varepsilon \rightarrow 0} \lim _{\delta \rightarrow 0} \iint_{A} \rho \ddot{u}_{i} u_{i, 1} d A-\int_{\Gamma} \frac{1}{2} \rho \dot{u}_{i} \dot{u}_{i} n_{1} d \Gamma
$$

In this manner, we get another form of $\mathrm{J}$ as follows

$$
\hat{J}=\lim _{\varepsilon \rightarrow 0} \lim _{\delta \rightarrow 0} \iint_{A}\left(\rho \ddot{u}_{i} u_{i, 1}-\rho \dot{u}_{i} \dot{u}_{i, 1}\right) d A+\int_{\Gamma}\left[\left(W_{e}+\frac{1}{2} \rho \dot{u}_{i} \dot{u}_{i}\right) n_{1}-T_{i} u_{i, 1}\right] d \Gamma
$$

As the integrand of the area integral has a singularity less than 2, the area integral is integrable. The three-dimensional $\hat{J}$ is constituted from the angle of dynamic stress intensity factor $K_{I}(t, v)$ in the form:

$$
\hat{J}=\frac{A_{I}(v) K_{I}^{2}(t)}{4 \mu\left(1-\kappa^{2}\right)}
$$

where

$$
\begin{gathered}
A_{I}(v)=\frac{2\left(1-\kappa^{2}\right) \beta_{0}^{2}\left(1-\kappa^{2} \beta_{0}^{2}\right)^{1 / 2}}{4\left[\left(1-\kappa^{2} \beta_{0}^{2}\right)\left(1-\beta_{0}^{2}\right)\right]^{1 / 2}-\left(2-\beta_{0}^{2}\right)^{2}} \\
\beta_{0}=v / c_{t} \\
\kappa=c_{t} / c_{1}
\end{gathered}
$$

$\mu$ means the shear modulus, $c_{t}$ the shear wave velocity, $c_{l}$ the longitudinal wave velocity.

In the case of a viscoelastic material, the close-to tip fields may be approximated by those of an elastic material with the elastic constants replaced by the initial values of the relaxation functions, because the strain rate is very high near the tip of a rapidly propagating crack. Therefore, replacement of $\mu$ by $\mu(0)$, the initial value of the relaxation function in shear (in eq. (17)) results in the relationship between $\hat{J}$ and $K_{I}(t, v)$ for a viscoelastic material.

The dynamic stress intensity factors for some problems can be calculated by applying the three apparently different expressions of the $\hat{J}$-integral (eqns (12), (14) and (16)). The conventional triangular constant strain elements are used and the crack node release technique is employed for modelling crack growth. The error due to the omission of the limiting processes may be estimated by comparing the results obtained from eqns (12) and (14) to those from eqn. (16).

The following problem is to be analyzed. A tensile step pulse $\sigma$ is applied to a centrally cracked rectangular plate (width $2 \mathrm{~W}=104 \mathrm{~mm}$, height $2 \mathrm{H}=40 \mathrm{~mm}$, 
initial crack length $2 \mathrm{a}=24 \mathrm{~mm}$, Poisson's ratio $\nu=0.286, \rho=2.45 \mathrm{Mp} / \mathrm{m}$, $\mu=29.4 \mathrm{GN} / \mathrm{m}$ ) at time $\mathrm{t}=0$. The crack is stationary for $\mathrm{t}<4.4 \mu \mathrm{s}$ and it begins to propagate at a constant velocity $v=1000 \mathrm{~m} / \mathrm{s}$. The $\hat{J}$-integral is computed over various determined trajectories, when selecting the paths which are to be translated as the crack tip approaches.

The $K_{I}(t, v)$ specified by the $\hat{J}$-integral is derived and the time when the nodal force starts to be released is indicated. It is observed that the dynamic stress intensity factor can be stipulated precisely without applying a special element.

\section{Conclusion}

To investigate the dynamic response of brittle materials weakened by sublevel crack aggregations, a simulation based on continuum damage mechanics was applied. The model uses the dynamic fracture process, which is a continuous increase in the damage when the damage is defined as the volume fraction of the material that has been tensile discharged by multiple crack growth and interaction. A numerical model of a center-cracked concrete sample under a step tension pulse was performed. Tension-softening characteristics in the concrete were predicted by the simulation to be the result of sublevel cracking effects that were present in the material, and not the assumed constitutive characteristics. The location of the damage region close to the crack is also predicted by the simulation. The local collapse from the view of the process zone and the global failure by virtue of the degree of reduction in load carrying capacity are also discussed. Analyses of accurate techniques for modelling crack growth with high acceleration or deceleration and those for simulating crack propagation in yielding materials would be necessary to assure a higher level of integrity and also safety of construction.

\section{Acknowledgement}

The author gratefully acknowledges financial support for this research from the Grant Agency of the Czech Republic (project No. 103/03/0655).

\section{References}

[1] Chen, E.P., Dynamic Brittle Fracture Analysis Based on Continuum Damage Mechanics. Fracture Mechanics: Perspectives and Directions (Twentieth Symposium), ASTM STP 1020, Philadelphia, pp. 447-458, 1989.

[2] Aoki, S., Dynamic crack problems. Topics in Engineering, Volume 16. In: Computational and Experimental Fracture Mechanics, CMP Southampton, pp. 111-138, 1991.

[3] Taylor, L.M. \& Flanagan, D.P., PRONTO 2D - A Two-Dimensional Transient Solid Dynamics Program. SAND 86-0594, Sandia National Laboratories Report, Albuquerque, NM, 1987. 